\title{
Abbreviations of Newspapers and Journals Cited
}

$B J R B$

$D G B$

$D Z J Y B$

$D Z R B$

FBIS

FJRB

GDNMB

$G M R B$

$G R B$

$G X R B$

GZRB

HBJJB

$H L B$

$J F R B$

$J J C K$

$J J R B$

$J N B$
Beijing ribao

Dagong bao (Beijing and Tianjin)

Dazhong jingying bao

Dazhong ribao

Foreign Broadcast Information

Service

Fujian ribao

Guangdong nongmin bao

Guangming ribao

Guangzhou ribao

Guangxi ribao

Guizhou ribao

Hebei jingii bao

Huili bao

Jiefang ribao

Jingii cankao

Jingii ribao

Jiangsu nongmin bao 
JPRS-CEA

JPRS-CPS

JPRS-CRA

JPRS-CRF

$M B R M B$

$N B R M B$

NCGZTX

NFRB

NMRB

NYJJWT

RMHYB

$R M R B$

SCMM

SCMP

SPRCM

STJP

SXNM

TKP

$X G S B$

$X H N B$

$X H S D X$

$Y R B$

ZGJJJB

ZGNMB

ZGQNB

ZJRB
Joint Publications Research

Service-China Report:

Economic Affairs

Joint Publications Research

Service-China Report: Political, Sociological, and Military Affairs

Joint Publications Research

Service-China Report: Agriculture

Joint Publications Research

Service-China: Red Flag

Minbei renmin bao

Nanbei renmin bao

Nongcun gongzuo tongxun

Nanfang ribao

Nongmin ribao

Nongye jingii wenti

Renmin hangyun bao

Renmin ribao

Survey of China Mainland

Magazines

Survey of China Mainland Press

Survey of the People's Republic of

China Magazines

Singtao Jibpao (Hong Kong)

Shanxi nongmin

Ta Kung Pao (Hong Kong)

Xianggang shibao

Xin Hunan bao

Xinbuashe dianxun

Yunnan ribao

Zhongguo jingii jibao

Zhongguo nongmin bao

Zhongguo qingnian bao

Zhejiang ribao 